\title{
Application of Atom Probe Tomography to Nitride Semiconductors
}

\author{
Rachel A. Oliver ${ }^{1}$, Fengzai Tang ${ }^{1}$,Samantha E. Bennett ${ }^{1}$, Tomas L. Martin ${ }^{2}$, Paul A.J. Bagot ${ }^{2}$, GDW \\ Smith $^{2}$, and Michael P. Moody ${ }^{2}$ \\ 1. Department of Materials Science and Metallurgy, University of Cambridge, 27 Charles Babbage Road, \\ Cambridge, CB3 0FS, United Kingdom \\ 2. Departments of Materials, University of Oxford, Parks Road, Oxford, OX1 3PH, United Kingdom
}

A decade ago, atom probe tomography (APT) was applied almost exclusively to the study of metals, since the study of materials with lower conductivities, such as semiconductors, was considered pragmatically to be very difficult. The advent of commercially-available laser-pulsed APT systems has since enabled the increasingly widespread application of APT to semiconductors, with particularly notable success in improving understanding of nitride materials.

The problem which first inspired the application of APT to the nitrides was the question of whether phase separation occurs in the InGaN quantum wells (QWs) which form the active region of blue and green light emitting diodes (LEDs). It had been suggested that indium-rich clusters a few nanometers in size in the InGaN QWs acted to localise charge carriers preventing their diffusion to defect sites, allowing impressive emission efficiencies to be achieved in materials with very high defect densities [1]. Evidence for this contention came from transmission electron microscopy (TEM) images, but the electron beam in the TEM had been seen damage the InGaN, leading to the observation of apparent indium-rich features which might not have been present in the as-grown material [2]. APT was seen as an ideal approach to investigate this question, since it utilises an entirely different imaging mechanism, provides direct compositional information about the sample and does not suffer from projection effects.

APT studies of blue- and green-emitting InGaN QWs did not show any evidence for the presence of non-random In-rich clusters, a significant but controversial result [3]. Many in the nitride community questioned the ability of APT to detect such clusters [4], but it was later shown that where the clusters were created in the TEM using an electron beam, they could be detected in APT [5]. Moreover, we have recently shown that although conventional QWs grown on the polar (0001) plane of GaN do not typically exhibit indium clustering, QWs grown on two non-polar planes - (1-100) and (11-20) (see Figure 1) - can exhibit a non-random indium distribution [6] with the degree of clustering being dependent not only on the growth plane but also the QW thickness and composition. Such studies not only validate the ability of APT to reveal clusters but also provide a vital insight into the structure of non-polar QWs and hence their optical properties and their potential usefulness in novel LEDs [7].

APT is now used to study the full range of nitride semiconductor alloys, including AlInN [8] and AlGaN [9] which have particularly wide bandgaps and low conductivities. In the growth of AlInN, the unintentional incorporation of gallium is a widespread problem, and APT provides a sensitive probe of the composition of the resulting quaternary alloy [8]. For both AlGaN and AlInN the detailed microstructure of high electron mobility transistors has also proved a fertile area of study [10]. Lastly, APT is increasingly used to address compositional variations around defects including inversion domains, stacking faults and dislocations (see Figure 2) [11]. Overall, in the decade since it was first applied to nitrides, APT has proved itself to be an extremely powerful technique for study of wide bandgap materials and has inspired the application of APT to other materials with limited conductivity. 
[1] Y Narukawa et al, Appl. Phys. Lett. 70 (1997) 981.

[2] TM Smeeton et al, Appl. Phys. Lett. 83 (2003) 5419.

[3] MJ Galtrey et al, J. Appl. Phys. 104 (2008) 013524.

[4] C Kisielowski and TP Bartel, Appl. Phys. Lett. 91 (2007) 176101.

[5] SE Bennett et al, Appl. Phys. Lett. 99 (2011) 021906.

[6] F. Tang et al, Appl. Phys. Lett. 106 (2015) 072104.

[7] S. Schulz et al, Phys. Rev. B 92 (2015) 235419.

[8] F. Tang et al, Microscopy and Microanalysis, 21 (2015) 544.

[9] SE Bennett et al, Ultramicroscopy 111 (2011) 207.

[10] B. Mazumder et al, Appl. Phys. Lett. 101 (2012) 091601.

[11] L Rigutti et al., Scripta Materialia (2017) In Press. DOI: 10.1016/j.scriptamat.2016.12.034
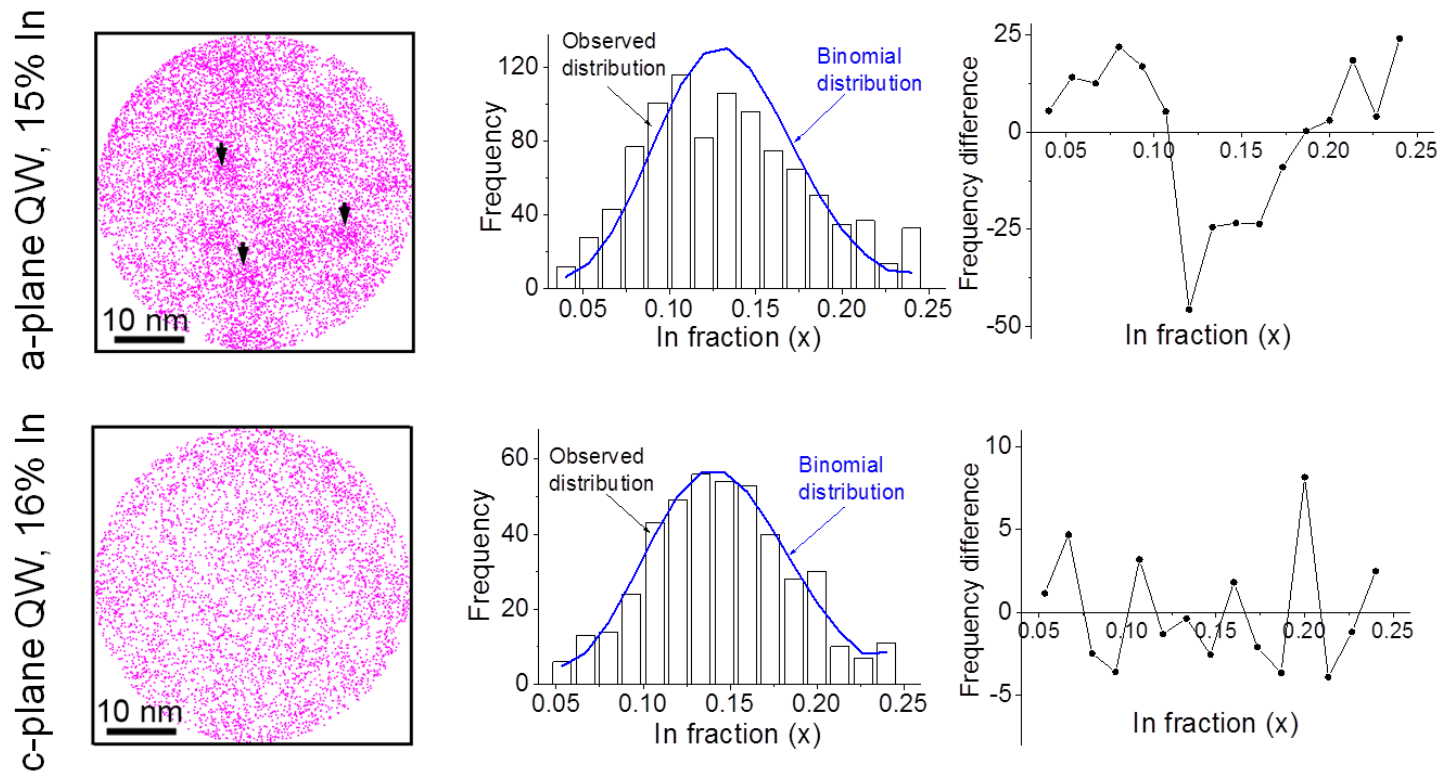

Figure 1. Comparison of APT analysis of c-plane (0001) oriented QW (bottom) and a-plane (1-100) oriented QW (top). Left: Plan view atom map of APT data from a single QW showing all In atoms but no $\mathrm{N}$ or Ga atoms. Middle: Frequency distribution histograms comparing observed variation in indium content to a binomial distribution. Right: Plots of the difference between the observed data and the binomial distribution.

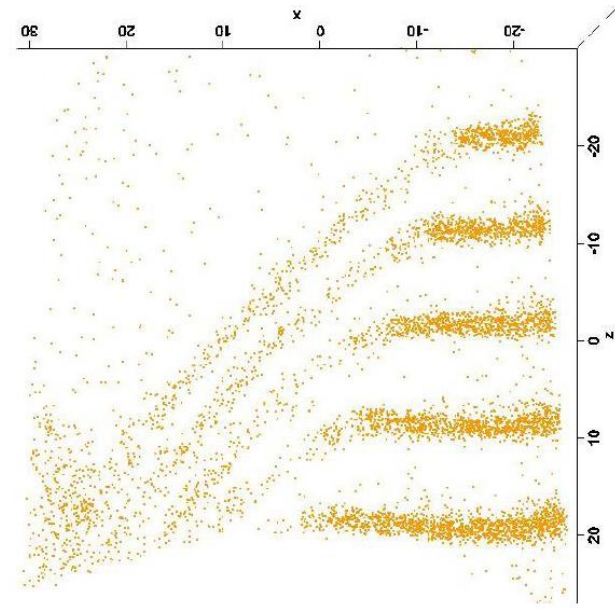

Figure 2. Atom map of an $\mathrm{InGaN} / \mathrm{GaN}$ multiple $\mathrm{QW}$ sample containing a dislocation showing all In atoms but no $\mathrm{N}$ or $\mathrm{Ga}$ atoms. The $\mathrm{x}$ and $\mathrm{z}$ scales are in $\mathrm{nm}$. The dislocation results in a V-pit at the sample surface which distorts the QWs. 